\title{
Assessment of the Global and Regional Land Hydrosphere and Its Impact on the Balance of the Geophysical Excitation Function of Polar Motion
}

\author{
Małgorzata WIŃSKA ${ }^{1}$, Jolanta NASTULA ${ }^{2}$, and Barbara KOŁACZEK ${ }^{2}$ \\ ${ }^{1}$ Warsaw University of Technology, Faculty of Civil Engineering, Warsaw, Poland \\ e-mail: m.winska@il.pw.edu.pl (corresponding author) \\ ${ }^{2}$ Space Research Center, Polish Academy of Sciences, Warsaw, Poland
}

\begin{abstract}
The impact of continental hydrological loading from land water, snow and ice on polar motion excitation, calculated as hydrological angular momentum (HAM), is difficult to estimate, and not as much is known about it as about atmospheric angular momentum (AAM) and oceanic angular momentum (OAM). In this paper, regional hydrological excitations to polar motion are investigated using monthly terrestrial water storage data derived from the Gravity Recovery and Climate Experiment (GRACE) mission and from the five models of land hydrology. The results show that the areas where the variance shows large variability are similar for the different models of land hydrology and for the GRACE data. Areas which have a small amplitude on the maps make an important contribution to the global hydrological excitation function of polar motion. The comparison of geodetic residuals and global hydrological excitation functions of polar motion shows that none of the hydrological excitation has enough energy to significantly improve the agreement between the observed geodetic excitation and geophysical ones.
\end{abstract}

Key words: polar motion, land hydrosphere models, GRACE data.

Ownership: Institute of Geophysics, Polish Academy of Sciences;

(C) 2015 Wińska et al. This is an open access article distributed under the Creative Commons Attribution-NonCommercial-NoDerivs license,

http://creativecommons.org/licenses/by-nc-nd/3.0/. 


\section{INTRODUCTION}

The Earth's rotation varies slightly with time, and these changes are both in the magnitude of the Earth's rotation and in the orientation of its axis. The magnitude of rotational rate change is measured by length-of-day variation. The orientation of the Earth's rotational axis with respect to the terrestrial reference frame is known as polar motion. The variable rotation is caused by mass redistribution and movement in the Earth system, which includes the atmosphere, ocean, land hydrology, and solid earth.

The changes in the global balance of the Earth's angular momentum due to non-tidal mass redistribution of geophysical fluids are still being studied to understand observed polar motion variations. Atmospheric and oceanic excitations of polar motion are mostly responsible for observed annual and semiannual wobble excitation (Gross 2005). The pressure term of the AAM variations is a major excitation for polar motion in the broad frequency band, but the motion term of the AAM is the dominant excitation source for the length of day variation. The motion component of the AAM is less important for polar motion than the pressure variation of the atmospheric angular momentum. The oceanic angular momentum is mostly driven by atmospheric forcing and thermohaline processes (Gross 2005).

Previous studies on the sum of global AAM and OAM excitation functions of polar motion bring the modelled excitation of polar motion closer to the observed one, but do not entirely explain the observed variations of polar motion which are determined by geodetic techniques (Brzeziński et al. 2005, 2009, Gross et al. 2003, Ponte et al. 1998, Nastula and Ponte 1999). The remaining can be accounted for by continental hydrological variations. Changes in water storage over land include changes of soil water, snow/ice sheets, and ground water. It has been assumed that hydrological angular momentum (HAM) variations over lands are not a negligible contributor to polar motion at seasonal timescales. The impact of continental hydrological signals on polar motion excitation is not known so well as the atmospheric and ocean ones. Determining HAM is difficult due to inadequate observational data and a hydrosphere that has not been thoroughly investigated.

In order to better understand the nature of the AAM and OAM signals, regional AAM and OAM variations were also previously investigated (Salstein and Rosen 1989, Nastula 1997, Nastula et al. 2000). The goal was to determine which regions in the ocean and atmosphere were acting as important sources of polar motion excitation. Regional analyses of the AAM and OAM have shown the importance of specific areas (e.g., the North Pacific and Southern Oceans for the OAM signals and Eurasia for the AAM signals) for polar motion excitation (Nastula et al. 2009). 
A number of previous studies have estimated hydrological excitation of polar motion by using climatological measurements (van Hylckama 1970, Hinnov and Wilson 1987, Chao and O'Connor 1988) and numerical climate models (Kuehne and Wilson 1991, Chen et al. 2000). Studies on hydrological excitation of polar motion were begun by van Hylckama (1970). These studies evolved in the late 1980s (Hinnov and Wilson 1987, Chao and Gross 1987, Kuehne and Wilson 1988, Chao and O'Connor 1988). Even then, large uncertainties and discrepancies existed among the conventional hydrological data sets as well as in hydrological modelling of the water budget. These studies on global hydrological excitation of polar motion adopted a certain runoff model and assumed its applicability to all regions on land. These models did not take into account water variations in hydrological reservoirs which have periods that last much longer than a month.

Recent studies have mostly focused on hydrological excitations of polar motion at seasonal timescales (Chen et al. 2000, Hopfner 2001, Nastula et al. 2008b). Now studies on hydrological excitation of polar motion are focused on excitations during seasonal and inter-seasonal timescales (Jin et al. 2010, Jin et al. 2012). The main aim of recent studies has been to better understand the effects of land hydrology on polar motion and to examine how the global budget of seasonal and inter-annual excitation of polar motion can be closed (Chen and Wilson 2005, Nastula et al. 2011, Jin et al. 2010, Jin et al. 2012). The impact of continental hydrological signals from land water, snow, and ice on polar motion excitation is not as well known as the atmospheric and ocean ones.

The hydrological angular momentum time series used in these studies were estimated from several models of global hydrology, such as: the Climate Prediction Center (CPC), Global Land Data Assimilation System (GLDAS), National Oceanic and Atmospheric Administration (NOAA), and Land Surface Discharge Model (LSDM).

The CPC model is computed based on the land data assimilation system (LDAS) models developed at NOAA Climate Prediction Center (CPC). LDAS is forced by observed precipitation derived from CPC daily and hourly precipitation analyses, downward solar and long-wave radiation, surface pressure, humidity, 2-m temperature, and horizontal wind speed from NCEP reanalysis. The output consists of soil temperature and soil moisture in four layers below the ground. At the surface, it includes all components affecting energy and water mass balance, including snow cover, depth, and albedo (Fan and van den Dool 2004).

The GLDAS model is determined on an $1^{\circ} \times 1^{\circ}$ latitude-longitude grid with monthly frequency in the period from January 1979 to present. The GLDAS generates optimal fields of land surface states and fluxes by integrating satellite and ground-based observational data products, using ad- 
vanced land surface modeling and data assimilation techniques (Rodell et al. 2004). The water storage is the sum of soil moisture, snow water equivalent, and canopy surface water, not counting changes in groundwater below the depth defined by the model.

The NOAA model is computed on the basis of the LadWorld global land dynamics model developed at the NOAA Geophysical Fluid Dynamics Laboratory. Simulated variables of this model include: snow water equivalent, soil water, shallow ground water, soil temperature, evapotranspiration, runoff stream flow, radiation, and latent heat fluxes.

The LSDM model simulates globally the vertical and lateral water transport and storage on land surfaces. The hydrological model captures all major continental water mass transport processes (soil moisture, snow, rivers and lakes, runoff, drainage) relevant for the generation of hydrological Earth rotation excitation functions (Walter 2008, Dill et al. 2009).

All of these hydrological models are available at the Special Bureau of Hydrology of the Global Geophysical Fluids Center (GGFC) of the International Earth Rotation and Reference Systems Service (IERS).

The models of global land hydrology give significantly different amplitudes and phases for polar motion excitation (Nastula et al. 2011, Chen and Wilson 2005). These models may not represent complete hydrological variation (Chen and Wilson 2005) because Terrestrial Water Storage (TWS) was not adequately measured at the continental scale (Lettenmaier and Famiglietti 2006).

Since 2002 the Gravity Recovery and Climate Experiment (GRACE) satellite mission has delivered precise time series of gravimetric variations and has allowed to determine the mass-gravimetric polar motion excitation function. Due to the GRACE mission, and to a lesser extent to the Laser Geodynamics Satellite (LAGEOS) missions, geophysical fluids' mass variability can be determined from gravity field observations. The coefficients of the second degree and of the first order of the Earth gravity field are proportional to variations of the equatorial components $\chi_{1}, \chi_{2}$ of the series of the gravimetric excitation function of polar motion. This gravimetric function can be compared with the mass term of geodetic excitation of polar motion. The gravimetric excitation functions' estimates from GRACE are subject to destriping, gridded mapping, and filter smoothing methods as well as aliasing errors (Jin et al. 2011). Several centers, such as the GeoForschungsZentrum (GFZ), the Center for Space Research (CSR), and the Jet Propulsion Laboratory (JPL), have computed the coefficients of series of time variable gravitation and of the adequate layer of water storage (Brzeziński et al. 2009, Chen and Wilson 2005, Seaone et al. 2009). In this study we used the GRACE data of RL04 and new RL05 series as computed by CSR. Major changes from the RL04 to the RL05 solutions include improved the knowledge of align- 
ments between the star camera, accelerometer, K-band ranging system for Level-1B data, and updated mean gravity field, ocean tide, pole tide, and dealiasing models for Level-2 processing. The CSR-RL05 field release consists of unconstrained gravity fields as well as a regularized gravity field timeseries that can be used for several applications without any post-processing error reduction (Chambers and Bonin 2012).

In papers by Kołaczek et al. (2011), Jin et al. (2010) and Jin et al. (2012), the global hydrological and gravimetric excitation functions of polar motion are compared with the residuals series GAM-AAM-OAM as computed by removing atmospheric (AAM) and oceanic (OAM) contributions from the GAM series (Nastula et al. 2011). In this study we used the geodetic time series obtained from the International Earth Rotation and Reference System Service (IERS) C04 series of polar motion (Bizouard and Gambis 2009). The atmospheric excitation functions AAM are derived from the time series of NCEP/NCAR reanalysis data (Salstein et al. 1993, Kalnay et al. 1996), ECMWF and ECMWF Era-Interim models (Dobslaw et al. 2010). The oceanic excitation functions OAM, including bottom pressure and currents term, were computed on the basis of the ECCO-JPL ocean model (Gross et al. 2003) and the OMCT model (Dobslaw et al. 2010).

Regional HAM based on models of global land hydrology and on GRACE data was also previously investigated (Nastula et al. 2008b, Nastula et al. 2008a, Nastula and Salstein 2012). Studies have shown similar geographic patterns for variances of regional excitation functions computed from models of land hydrology and from the GRACE data. The studies show that the strongest values are in the following regions: Southeast and South Asia, the Amazon basin of South America, the southeast United States, and areas north of the Mediterranean Sea. Additionally, these studies have shown that the GRACE regional excitation functions of polar motion have larger magnitudes than the regional hydrological excitation functions computed from hydrological models.

The main purpose of our present paper was to carefully study the regional hydrological contribution to polar motion excitations and to compare regional hydrological excitations on polar motion based on different models of land hydrology and on GRACE data.

In this study we analysed the following issues: first, we computed the regional distribution of hydrological and gravimetric excitation functions of polar motion, and we determined the regions in which these functions were reaching maxima values; next, we computed and compared the time series corresponding to these regions; finally, we compared global and regional excitation functions with geodetic residuals. 


\section{ANALYSIS AND RESULTS}

\subsection{Regional patterns}

Hydrological and gravimetric excitations of polar motion were computed based on the following formulas (Eubanks 1993):

$$
\left[\begin{array}{l}
\chi_{1} \\
\chi_{2}
\end{array}\right]=-\frac{1.098 R_{e}^{2}}{C-A} \iint \Delta q(\varphi, \lambda, t) \sin (\varphi) \cos (\varphi)\left[\begin{array}{c}
\cos (\lambda) \\
\sin (\lambda)
\end{array}\right] d S,
$$

where $\Delta q(\varphi, \lambda, t)$ represent the changes in water storage in unit area (in $\left.\mathrm{kg} / \mathrm{m}^{2}\right), R_{e}$ is the Earth's mean radius, $d S$ is the surface element area, and $C$ and $A$ are the Earth's principal moments of inertia. The factor 1.098 accounts for the combined effects of the yielding of the solid Earth to the surface load, core-mantle decoupling and rotational deformation (Eubanks 1993).

Changes of terrestrial water storage $\Delta q(\varphi, \lambda, t)$ for hydrological excitation functions are given directly in available models of land hydrosphere.

Changes of terrestrial water storage $\Delta q(\varphi, \lambda, t)$ based on the gravity data were computed from the spherical harmonic coefficients of the Earth's gravity field (Wahr and Molenaar 1998):

$$
\Delta \bar{\sigma}(\varphi, \lambda)=\frac{2 \pi a \rho_{\text {ave }}}{3} \sum_{l=0}^{L} \frac{2 l+1}{1+k_{l}} W_{l} \sum_{m=0}^{l} \bar{P}_{l m}(\sin \varphi) \cdot\left(\Delta \bar{c}_{l m} \cos (m \lambda)+\Delta \bar{s}_{l m} \sin (m \lambda)\right),
$$

where $a$ is the radius of the Earth, $\bar{P}_{l m}$ is the normalized associated Legendre function, $\rho_{\text {ave }}$ is the average density of the Earth, $\Delta \bar{c}_{l m}$ and $\Delta \bar{s}_{l m}$ are the dimensionless coefficients, and $W_{l}$ is an averaging function.

All of these considered data series are available with a resolution of one month. The common time span for all considered data covered years from 2003.0 to 2006.0 .

Figures 1 and 2 compare the geographic variability of components $\chi_{1}$ and $\chi_{2}$ of the hydrological and gravimetric excitation functions estimated from different models of land hydrology and from the GRACE data. One can see that the areas where the variance shows large variability are generally similar for the different models of land hydrology and for the GRACE data. These areas are: the west coast of North America, the Brazilian Highlands, South Africa and Europe for the $\chi_{1}$ component, and the Mississippi basin, the Great Lakes region of North America, the Brazilian Highlands, and the southern region of the Asian continent for the $\chi_{2}$ component.

One exception is the variance change estimated from the LSDM model. In this case, maps of the variance show different geographical patterns and have smaller amplitudes than those obtained from the other models. This is perhaps due to the fact that the LSDM model captures all major continental 

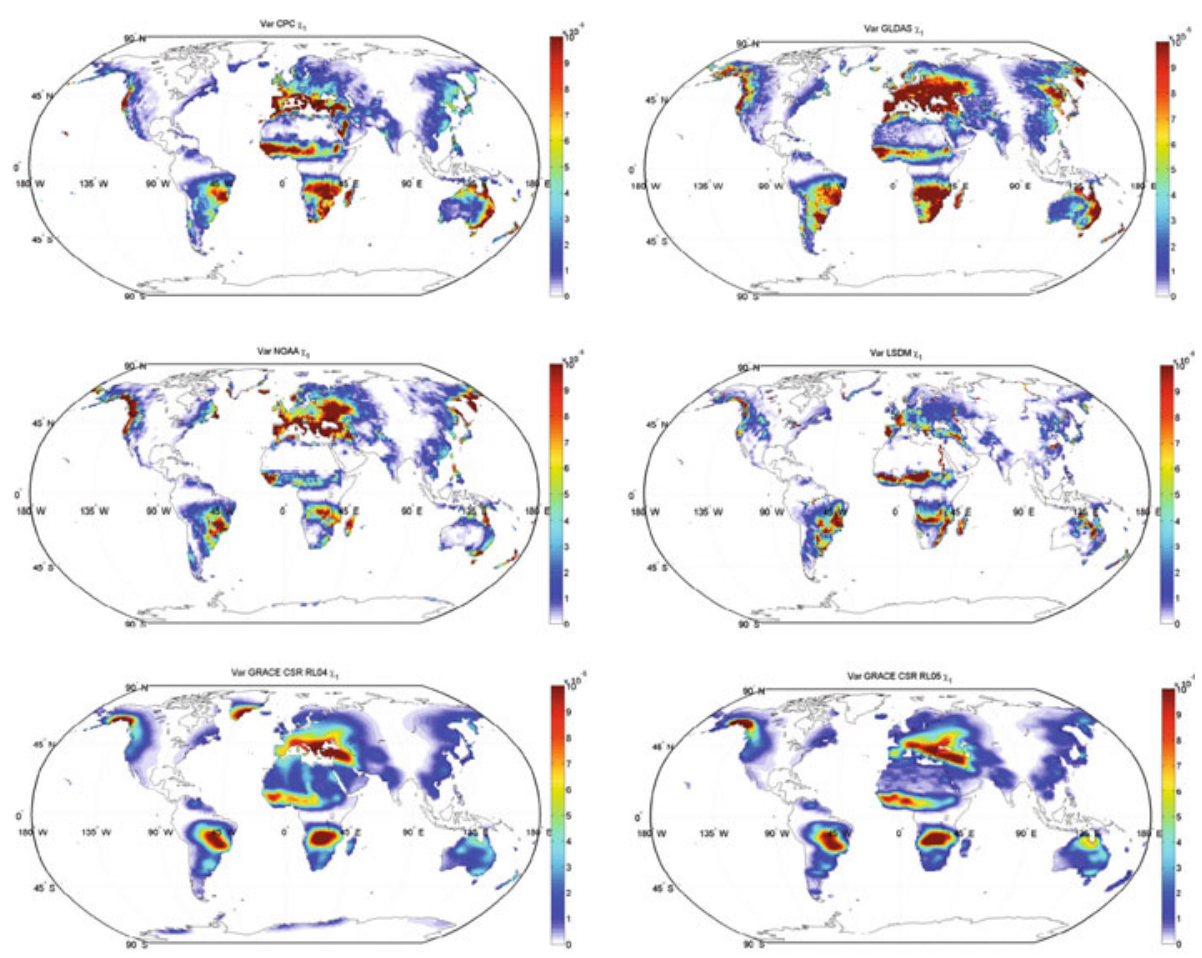

Fig. 1. Maps of variances of the $\chi_{1}$ component of the regional hydrological and gravimetric excitation functions of polar motion computed from different models of land hydrosphere and from the GRACE satellite mission. Units are mas ${ }^{2}$.

water mass transport processes (soil moisture, snow, rivers and lakes, runoff, drainage) relevant for the generation of hydrological Earth rotation excitation functions (Walter 2008, Dill et al. 2009). It should be noted, however, that between patterns of the $\chi_{2}$ component computed from the different models of land hydrology, there exist some discrepancies; namely, variances of the $\chi_{2}$ component of the hydrological excitation function of the CPC model do not show changeability in the West Siberian Plain and in the Central Siberian Plateau.

The maximum changes of variances are in the order of $10^{-4} \mathrm{mas}^{2}$ for both the $\chi_{1}$ and $\chi_{2}$ component. One can see that the regional $\chi_{2}$ component shows changes in larger areas of lands than the $\chi_{1}$ component. These regional patterns differences might be caused by using different number of input variables in the hydrological models that are given in Table 1. 

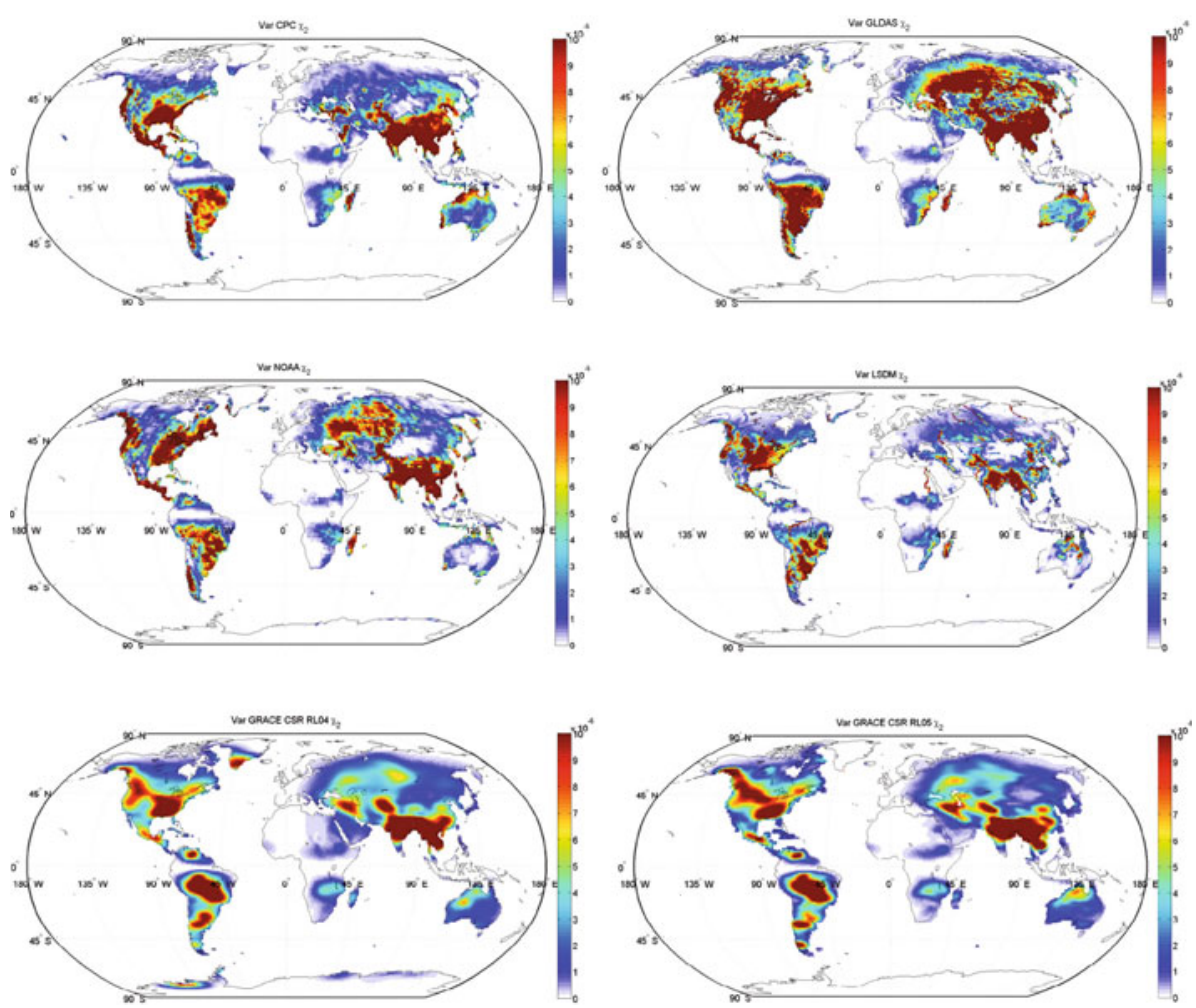

Fig. 2. Maps of variances of the $\chi_{2}$ component of the regional hydrological and gravimetric excitation functions of polar motion computed from different models of land hydrosphere and from the GRACE satellite mission. Units are mas².

\subsection{Time series of regional excitation functions}

In order to compare regional changes of the excitation functions in a quantitative manner we chose to further analyses several regions based on visual inspection of the maps. The boundaries of the selected areas of maximum variability of regional excitation functions are shown in Table 2. Time series of contributions to the hydrological and gravimetric excitation functions from five selected areas were computed by integration of the formula given by Eq. 1 within the given boundaries.

Figure 3 shows the time series of regional excitations estimated for five different land areas of maximum variability, as described in Table 2, from the four land hydrological models (CPC, GLDAS, NOAA, and LSDM), and from two GRACE data series (CSR RL04 and CSR RL05). Figure 4 and Table 4 , both of which contain the standard deviations, show regional differences in the results obtained from the different models. It can be seen that 
Table 1

The input variables of land hydrological models that are taken into account when HAM functions are computed from different land hydrological models

\begin{tabular}{|l|c|c|c|c|}
\hline \multicolumn{1}{|c|}{ Variables/models } & CPC & GLDAS & NOAA & LSDM \\
\hline Snow water equivalent & $\times$ & $\times$ & $\times$ & $\times$ \\
Total precipitation & $\times$ & $\times$ & & $\times$ \\
Soil moisture & $\begin{array}{c}\text { 4 layer below } \\
\text { the ground }\end{array}$ & $\begin{array}{c}\text { 4 layer below } \\
\text { the ground }\end{array}$ & $\times$ & $\times$ \\
Evapotranspiration & & $\times$ & $\times$ & $\times$ \\
Total runoff & & & $\times$ & $\times$ \\
Surface water (river, lakes) & & & $\times$ & $\times$ \\
Ground water & & & $\times$ & \\
Drainage & & & $\times$ & \\
Radiation & & & & \\
Latent heat flux & & & & \\
Soil temperature & & & $\times$ & \\
Swamps & & & & \\
Canopy surface water & & & & \\
Greenland ice & & & & \\
\hline
\end{tabular}

Note: None of these models includes information of Terrestrial Water Storage in Antarctica ice.

Table 2

Selected areas where regional hydrological and gravimetric excitation functions reach and achieve maximum values

\begin{tabular}{|l|c|c|}
\hline \multicolumn{1}{|c|}{ Area } & Latitude & Longitude \\
\hline Europe & $30^{\circ} \mathrm{N}-55^{\circ} \mathrm{N}$ & $0^{\circ} \mathrm{E}-45^{\circ} \mathrm{E}$ \\
Asia & $0^{\circ} \mathrm{N}-55^{\circ} \mathrm{N}$ & $60^{\circ} \mathrm{E}-180^{\circ} \mathrm{E}$ \\
Africa & $45^{\circ} \mathrm{S}-0^{\circ}$ & $15^{\circ} \mathrm{E}-90^{\circ} \mathrm{E}$ \\
North America & $26^{\circ} \mathrm{N}-50^{\circ} \mathrm{N}$ & $55^{\circ} \mathrm{W}-110^{\circ} \mathrm{W}$ \\
South America & $45^{\circ} \mathrm{S}-0^{\circ}$ & $150^{\circ} \mathrm{W}-180^{\circ} \mathrm{W}$ \\
\hline
\end{tabular}

Note: HAM Maxima Sums for both the $\chi_{1}$ and $\chi_{2}$ components were calculated only for these regions.

the largest amplitudes of the $\chi_{1}$ component of hydrological excitation are for the European region, and for the $\chi_{2}$ components they are for the Asian region. The smallest amplitudes of the $\chi_{1}$ components are for the North American regions, and the $\chi_{2}$ components are for the African regions. The model- 


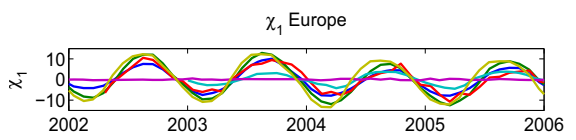

$\chi_{1}$ South America

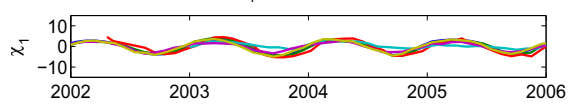

$\chi_{1}$ North America

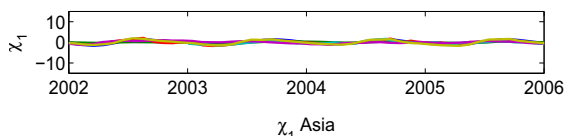

$\chi_{1}$ Asia

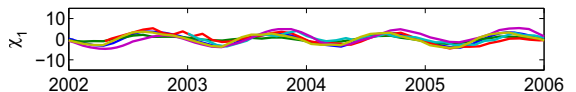

$\chi_{1}$ Africa

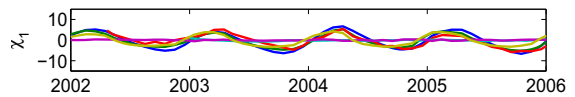

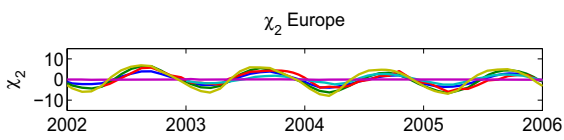

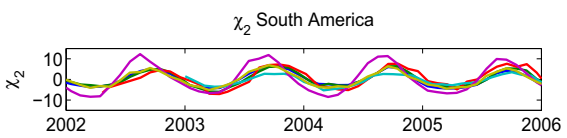

$\chi_{2}$ North America

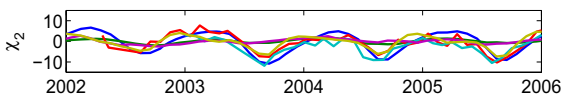

$\chi_{2}$ Asia

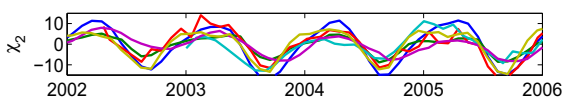

$\chi_{2}$ Africa

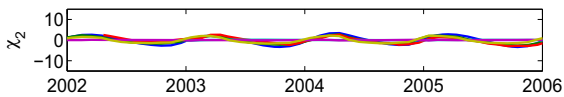

- CPC — GLDAS — GRACE CSR RL04 — GRACE CSR RL05 — LSDM — NOAA

Fig. 3. Variations of the hydrological and gravimetric excitation functions of polar motion, $\chi_{1}$ and $\chi_{2}$, computed for selected land areas of maximum variability shown in Table 2 . The mean and trend were removed. The units of $\chi_{1}$ and $\chi_{2}$ are mas.
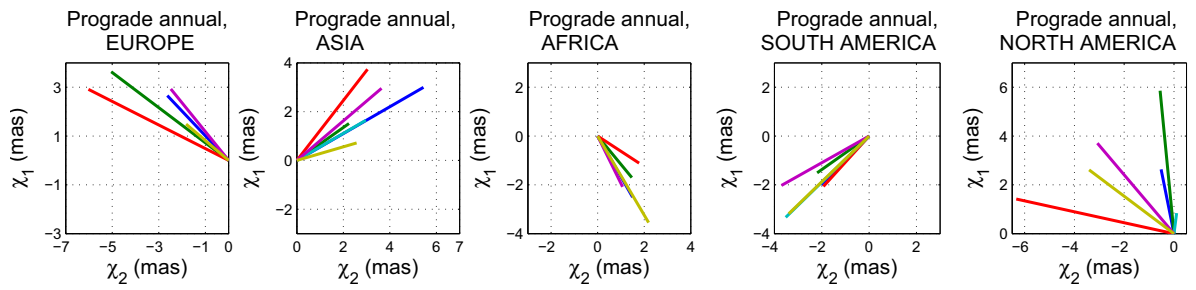

— CPC — GLDAS — NOAA — LSDM — GRACE CSR RL04 — GRACE CSR RL05

Retrograde annual,

Retrograde annual,
ASIA

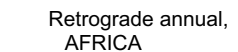

Retrograde annual,
SOUTH AMERICA

Retrograde annual,
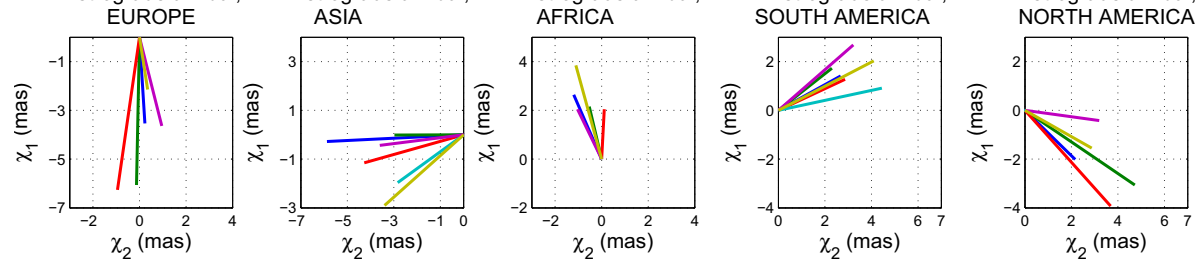

Fig. 4. Phasor diagrams of annual variations of the complex-valued component $\chi_{1}+i \chi_{2}$ of the regional hydrological and gravimetric excitation function. Analysis was done over the period of 2003.0-2006.0. 
Table 3

Standard deviations of regional hydrological and gravimetric excitations of polar motion being a sum of the most variable excitations in the five regions taken into consideration

\begin{tabular}{|c|c|c|c|c|c|c|c|c|c|c|}
\hline \multicolumn{11}{|c|}{ Standard deviations of regional HAM } \\
\hline \multirow{2}{*}{$\begin{array}{l}\text { Region } \\
\text { Model }\end{array}$} & \multicolumn{2}{|c|}{ Africa } & \multicolumn{2}{|c|}{$\begin{array}{c}\text { South } \\
\text { America }\end{array}$} & \multicolumn{2}{|c|}{ Asia } & \multicolumn{2}{|c|}{ Europe } & \multicolumn{2}{|c|}{$\begin{array}{c}\text { North } \\
\text { America }\end{array}$} \\
\hline & $\begin{array}{c}\chi_{1} \\
{[\mathrm{mas}]}\end{array}$ & $\begin{array}{c}\chi_{2} \\
{[\mathrm{mas}]}\end{array}$ & $\begin{array}{c}\chi_{1} \\
{[\mathrm{mas}]}\end{array}$ & $\begin{array}{c}\chi_{2} \\
{[\mathrm{mas}]}\end{array}$ & $\begin{array}{c}\chi_{1} \\
{[\mathrm{mas}]}\end{array}$ & $\begin{array}{c}\chi_{2} \\
{[\mathrm{mas}]}\end{array}$ & $\begin{array}{c}\chi_{1} \\
{[\mathrm{mas}]}\end{array}$ & $\begin{array}{c}\chi_{2} \\
{[\mathrm{mas}]}\end{array}$ & $\begin{array}{c}\chi_{1} \\
{[\mathrm{mas}]}\end{array}$ & $\begin{array}{c}\chi_{2} \\
{[\mathrm{mas}]}\end{array}$ \\
\hline $\mathrm{CPC}$ & 4.03 & 2.08 & 2.58 & 3.48 & 2.30 & 8.76 & 5.13 & 2.31 & 1.91 & 4.90 \\
\hline GLDAS & 3.34 & 1.66 & 2.76 & 3.90 & 1.11 & 4.35 & 7.43 & 3.58 & 1.14 & 0.57 \\
\hline CSR RL04 & 3.31 & 1.68 & 3.69 & 5.53 & 2.35 & 6.99 & 2.95 & 5.56 & 1.12 & 4.71 \\
\hline CSR RL05 & 0.01 & 0.00 & 1.51 & 1.84 & 1.87 & 6.15 & 0.02 & 0.01 & 1.00 & 4.52 \\
\hline LSDM & 0.23 & 0.09 & 1.79 & 6.56 & 3.39 & 5.38 & 0.22 & 0.09 & 0.09 & 0.53 \\
\hline NOAA & 2.73 & 1.38 & 2.65 & 3.60 & 2.29 & 6.87 & 8.40 & 4.53 & 0.78 & 3.43 \\
\hline
\end{tabular}

led hydrological and gravimetric time series of the $\chi_{1}$ components for the European region varied from the mean on the 3-8 mas level, apart from the time series based on the LSDM model and the GRACE CSR RL05 data. The time series of the $\chi_{2}$ components for the Asian region varied from the mean on the 5-9 mas level (see Table 3). The described differences are quite natural, due to geographic position of these regions, since the $x$-axis is directed approximately towards Europe and Africa, while the $y$-axis directs roughly towards Asia and the Americas.

A visual inspection of Fig. 3 clearly shows that an annual oscillation is the dominant signal in the regional excitation functions of polar motion (Fig. 3). Therefore, we decided to compare the annual oscillation parameters for the five regions of maximum variability and for different hydrological and gravimetric excitation functions. To do so, we computed the prograde and retrograde terms of the annual signals of the complex component $\chi_{1}+i \chi_{2}$ for each series shown in Fig. 3 by using the least-squares method (Brzeziński 1992).

The phasor plots of different regional hydrological and gravimetric excitations are shown in Fig. 4. The phases and amplitudes of annual oscillation are compatible with one another within the same region. The largest agreement exists for phases of prograde annual oscillation in the South American region and for the retrograde annual component in the African region. The largest discrepancies exist for the amplitudes of the prograde annual oscillations in the North American region. Vectors of prograde annual oscillation of the European area of maximum variability are shifted in the direction of 
Table 4

Standard deviations of global (HAM Global) and regional HAM Maxima Sum variations and of the differences between the HAM Global and HAM Maxima Sum excitation functions and of three kinds of geodetic residuals

\begin{tabular}{|c|c|c|}
\hline \multicolumn{3}{|c|}{ HAM global } \\
\hline Data & $\chi_{1}[\mathrm{mas}]$ & $\chi_{2}$ [mas] \\
\hline $\mathrm{CPC}$ & 3.92 & 10.74 \\
\hline GLDAS & 5.66 & 13.95 \\
\hline GRACE CSR RL04 & 8.79 & 8.43 \\
\hline GRACE CSR RL05 & 7.25 & 10.96 \\
\hline LSDM & 4.66 & 10.67 \\
\hline NOAA & 4.70 & 5.82 \\
\hline \multicolumn{3}{|c|}{ HAM Maxima Sum } \\
\hline Data & $\chi_{1}[\mathrm{mas}]$ & $\chi_{2}$ [mas] \\
\hline $\mathrm{CPC}$ & 2.70 & 5.41 \\
\hline GLDAS & 4.00 & 2.94 \\
\hline GRACE CSR RL04 & 4.36 & 5.39 \\
\hline GRACE CSR RL05 & 1.83 & 6.89 \\
\hline LSDM & 2.28 & 4.54 \\
\hline NOAA & 5.66 & 3.02 \\
\hline \multicolumn{3}{|c|}{ Residuals = HAM global - HAM Maxima Sum } \\
\hline Data & $\chi_{1}[\mathrm{mas}]$ & $\chi_{2}[\mathrm{mas}]$ \\
\hline $\mathrm{CPC}$ & 3.34 & 5.51 \\
\hline GLDAS & 5.04 & 12.29 \\
\hline GRACE CSR RL04 & 5.43 & 5.61 \\
\hline GRACE CSR RL05 & 6.59 & 7.42 \\
\hline LSDM & 3.26 & 6.29 \\
\hline NOAA & 5.19 & 4.05 \\
\hline \multicolumn{3}{|c|}{ Geodetic residuals } \\
\hline Data & $\chi_{1}[\mathrm{mas}]$ & $\chi_{2}[\mathrm{mas}]$ \\
\hline GAM-ECMWF-OMCT & 15.17 & 18.18 \\
\hline GAM-ERA-Interim-OMCT & 14.67 & 18.31 \\
\hline GAM-NCEP/NCAR-ECCO & 12.32 & 12.94 \\
\hline \multicolumn{3}{|c|}{ Root mean square value of AAM and OAM models } \\
\hline Data & $\chi_{1}[\mathrm{mas}]$ & $\chi_{2}[\mathrm{mas}]$ \\
\hline ECMWF & 30.51 & 42.18 \\
\hline NCEP/NCAR & 24.57 & 34.38 \\
\hline Era-Interim & 29.67 & 35.09 \\
\hline ECCO & 13.21 & 20.51 \\
\hline OMCT & 28.46 & 28.16 \\
\hline
\end{tabular}



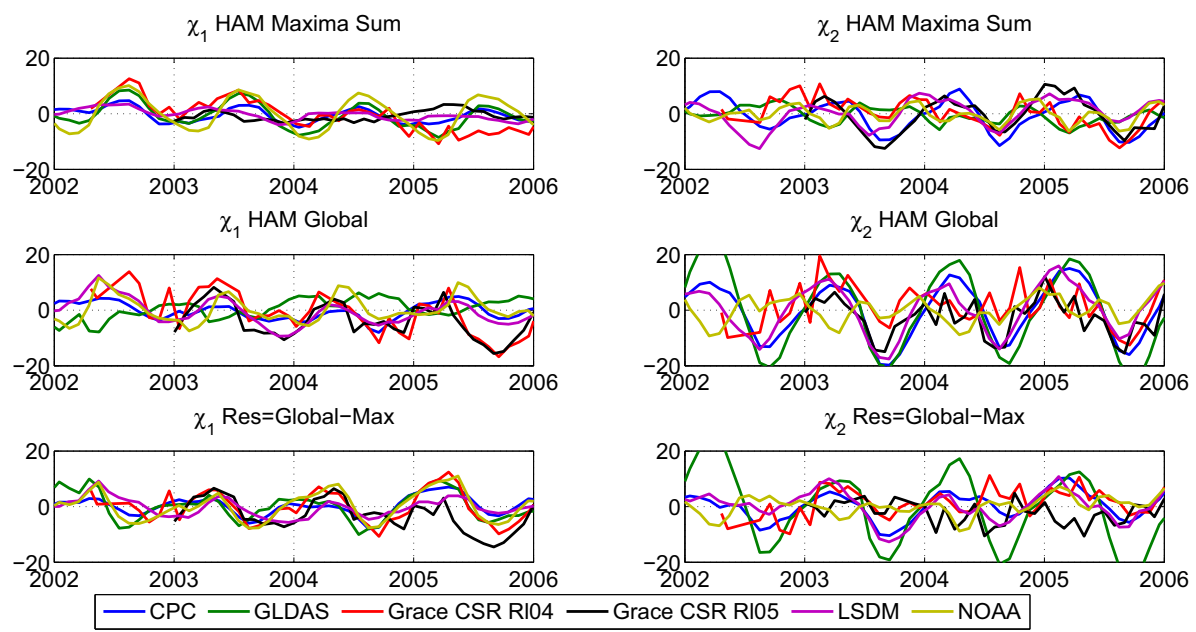

Fig. 5. Variations of hydrological and gravimetric excitation functions of polar motion, $\chi_{1}$ and $\chi_{2}$. The upper panels present the HAM Maxima Sum excitation function calculated from the sum of the areas of maximum variability shown in Table 2 . The middle panels present the global HAM excitation functions computed from all land areas. The bottom panels present residuals being the differences between the global HAM excitation functions and the HAM Maxima Sum excitation functions. The mean and trend were removed from the time series. The units of $\chi_{1}$ and $\chi_{2}$ are mas.

about 90 degrees relative to the prograde vectors of the Asian area. Similarly, the prograde vectors of the South American area of maximum variability are displaced in relation to the vectors of the African area by about 90 degrees, whereas in the opposite phase the following pairs of prograde annual oscillation, i.e., European and South American areas of maximum variability and Asian and African areas of maximum variability are. In the case of retrograde annual oscillations, the following pairs of vectors are directed in the opposite direction: European and African, Asian and South American. Annual oscillation of the North American area of maximum variability has a direction that is roughly similar to the direction of annual oscillation of the European area in both the prograde and retrograde parts.

Next, regional excitation functions, being the sum of contributions of chosen regions of maximum variability (HAM Maxima Sum), and global excitation functions (HAM Global), being the sum of all contributions of land areas, were computed from four land hydrological models - CPC, GLDAS, NOAA, and LSDM, and from two GRACE data series - CSR RL04 and CSR RL05. Figure 5 shows the comparison of time series of the sum of maximum areas (HAM Maxima Sum), the sum of all land areas (HAM Global), and their differences $($ Res $=$ Global - Max $)$. The means and 
linear trend are removed from all data sets. From a visual inspection one can see that the regional hydrological and gravimetric excitation functions of polar motion (HAM Maxima Sum) shown in the top panel are not sufficiently consistent with one another, i.e., they differ in their amplitudes and phases. The spread of these amplitudes is also confirmed by the results in Table 4, where the standard deviations of the HAM Maxima Sum functions are given; for example, for the $\chi_{1}$ component the standard deviation has a value of from 1.8 to $5.7 \mathrm{mas}$, and for the $\chi_{2}$ component the standard deviation has a value of from 2.9 to 6.9 mas. We also observed that regional hydrological and gravimetric excitation functions are dominated by annual fluctuations, in which the fluctuations for the $\chi_{1}$ component have smaller amplitudes than for the $\chi_{2}$ component.

It should be noted that the HAM Maxima Sum functions have quite comparable amplitudes to the global HAM Global functions (middle panel of Fig. 5) in the case of the $\chi_{1}$ component, while in case of $\chi_{2}$ component the HAM Maxima Sum functions have smaller amplitudes than the global HAM Global functions (Fig. 5). Standard deviations of HAM Global, as shown in Table 3, are larger than those of the HAM Maxima Sum for both the $\chi_{1}$ and $\chi_{2}$ components. The time series of the residuals $(\mathrm{Res}=\mathrm{Global}-\mathrm{Max})$ both for $\chi_{1}$ and $\chi_{2}$ have variations that are comparable with the HAM Maxima Sum (Fig. 3). The standard deviations of the residuals (Res = Global - Max), although they are generally smaller than the standard variations of global HAM, are generally larger than the standard variations of the global HAM Maxima Sum (Table 4). For the GLDAS model, the residuals for the $\chi_{2}$ component are even much larger than both the HAM Maxima Sum and global HAM functions. The exceptions are the standard deviations obtained from the NOAA model, whose value for the $\chi_{2}$ component of the residuals is smaller than the value for $\chi_{2}$ of the HAM Maxima Sum (Table 4). The above considerations showed that areas which were not taken into account in the HAM Maxima Sum solutions make an important contribution to the HAM Global function, even if they have a small amplitude on the maps.

\subsection{Comparison of global excitation functions with geodetic residuals}

Finally, we decided to compare the global HAM Global functions with the geodetic excitation function in order to check which of these hydrological and gravimetric functions gives a result that is closest to that obtained from the geodetic observations. In order to estimate a hydrological signal in the observed, geodetic excitation function, we computed the differences between the geodetic excitation function of polar motion GAM (Geodetic Angular Momentum) and the joint atmospheric plus oceanic excitation functions (Atmospheric Angular Momentum + Oceanic Angular Momentum - AAM+ 
(a)
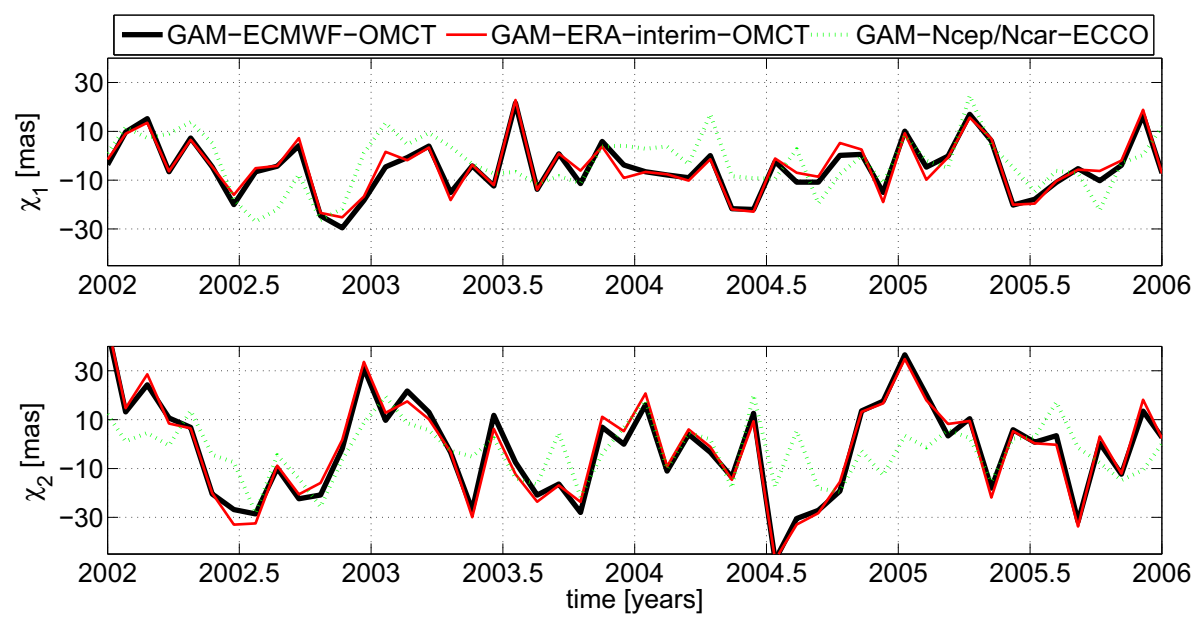

(b)
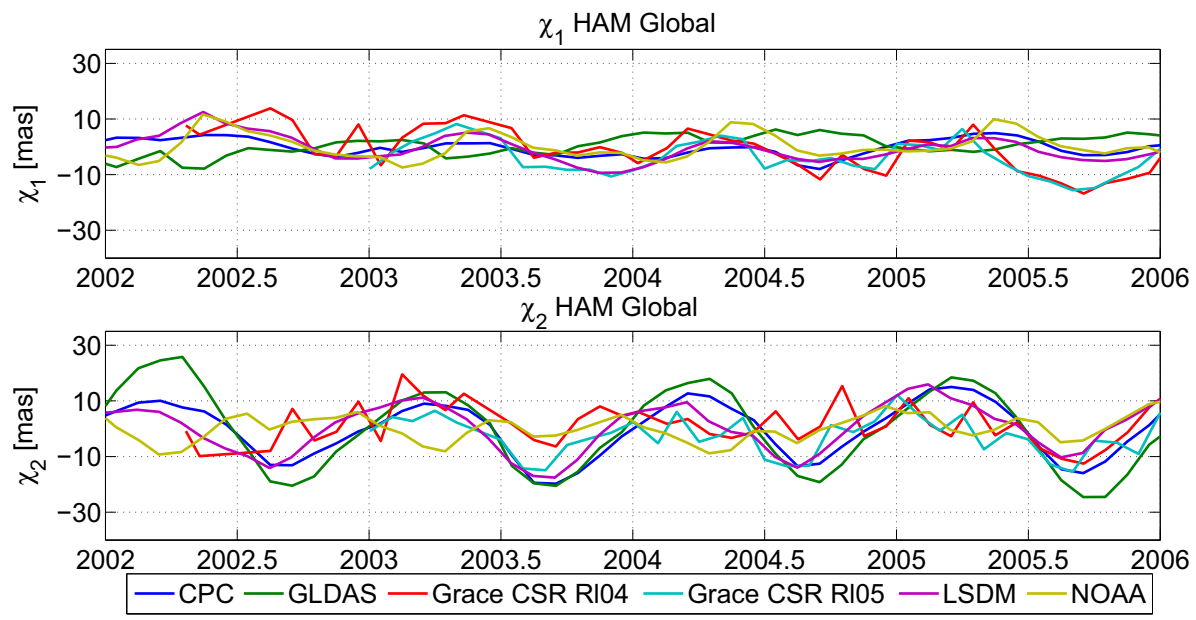

Fig. 6: (a) Geodetic residuals $\chi_{1}$ and $\chi_{2}$ obtained from different models of atmospheric and oceanic excitations of polar motion: GAM-ECMWF-OMCT, GAM-ERAInterim-OMCT, and GAM-NCEP/NCAR-ECCO; (b) global gravimetric and hydrological excitation functions of polar motion, $\chi_{1}$ and $\chi_{2}$. The mean and trend were removed from the time series.

OAM), hereinafter called geodetic residuals (Fig. 6). The observed geodetic excitation functions were derived from the Earth Rotation Parameters combined series C04 by the IERS Earth Orientation Center (http://hpiers.obspm. fr/eop-pc/index.php?index=excitactive\&lang=en). 
The geodetic residuals describe approximately the hydrological signals in the geodetically-based polar motion excitation function; however, they still contain not modelled signals from the change in mass of the ice sheet, and the effects of earthquakes. In order to determine the residuals one needs a correct model of the atmosphere (AAM) and ocean (OAM). Obviously, errors in these models also affect the resulting geodetic residuals. Here the geodetic residuals were computed based on the three different atmospheric models (ECMWF, NCEP/NCAR, Era-Interim) and two different oceanic models (ECCO, OMCT). The resulting geodetic residuals are shown in Fig. 6a. We should emphasize that significant differences (between 1020 mas) occurred between the geodetic residuals determined using different oceanic models. In contrast, the use of different atmospheric models did not cause any significant difference in the geodetic residuals. The standard deviation determined for these geodetic residuals was the least for the GAMNCEP/NCAR-ECCO series and was at the 12 mas level for both the $\chi_{1}$ and $\chi_{2}$ component, where the standard variation for other residuals is at a higher level (see Table 3). The geodetic residuals computed for the ECCO and NCEP/NCAR models had the smallest amplitude. Our results show that the impact of the oceans on polar motion excitation is still not well estimated, mainly due to difficulties in modelling mass redistribution over the ocean.

As is shown in Table 5 and Fig. 6, generally there are fewer variations of the HAM Global than variations of the geodetic residuals. This proves that the HAM excitation functions do not sufficiently explain the considered geodetic residuals in the seasonal scale.

Table 5

Correlation coefficients between global geodetic and geophysical excitation functions of polar motion, $\chi_{1}$ and $\chi_{2}$, calculated after removing annual signals from the time series; geodetic residuals were calculated as differences between

GAM (C04 series) and the sum of AAM (the NCEP/NCAR model was used) and OAM (the ECCO model was used)

\begin{tabular}{|l|c|c|}
\hline \multicolumn{3}{|c|}{ Correlation coefficients } \\
\hline Geodetic residuals versus & $\chi_{1}[\mathrm{mas}]$ & $\chi_{2}[\mathrm{mas}]$ \\
\hline CPC & 0.22 & 0.29 \\
GLDAS & 0.12 & 0.24 \\
GRACE CSR RL04 & 0.07 & 0.21 \\
GRACE CSR RL05 & 0.12 & 0.53 \\
LSDM & 0.29 & 0.40 \\
NOAA & 0.24 & 0.25 \\
\hline
\end{tabular}

Note: statistical significance $p=0: 3$. 
Table 6

Amplitudes and phases of the prograde and retrograde annual oscillations of the different hydrological and gravimetric excitation functions using Last Square Method and of the residuals of the geodetic excitation function (G-A-O)

\begin{tabular}{|l|c|r|r|r|}
\hline \multirow{2}{*}{\multicolumn{1}{|c|}{ Model }} & \multicolumn{2}{c|}{ Prograde } & \multicolumn{2}{c|}{ Retrograde } \\
\cline { 2 - 5 } & $\begin{array}{c}\text { Amplitude } \\
{[\mathrm{mas}]}\end{array}$ & $\begin{array}{c}\text { Phase } \\
{\left[{ }^{\circ}\right]}\end{array}$ & $\begin{array}{c}\text { Amplitude } \\
{[\mathrm{mas}]}\end{array}$ & $\begin{array}{c}\text { Phase } \\
{\left[{ }^{\circ}\right]}\end{array}$ \\
\hline CPC & $2.69 \pm 0.19$ & $45.15 \pm 4.50$ & $2.95 \pm 0.19$ & $-143.01 \pm 3.78$ \\
GLDAS & $8.34 \pm 0.49$ & $-166.55 \pm 3.34$ & $8.86 \pm 0.57$ & $-42.68 \pm 3.70$ \\
GRACE CSR RL04 & $4.82 \pm 0.66$ & $129.71 \pm 7.86$ & $3.22 \pm 0.52$ & $-7.44 \pm 9.41$ \\
GRACE CSR RL05 & $2.77 \pm 1.04$ & $-40.41 \pm 21.49$ & $6.09 \pm 1.02$ & $121.73 \pm 9.56$ \\
LSDM & $3.42 \pm 0.14$ & $82.99 \pm 18.65$ & $3.49 \pm 0.17$ & $142.51 \pm 20.11$ \\
NOAA & $6.49 \pm 0.16$ & $167.39 \pm 1.43$ & $5.38 \pm 0.19$ & $-46.81 \pm 2.04$ \\
G-A-O & $5.32 \pm 0.55$ & $-43.45 \pm 4.25$ & $3.75 \pm 0.48$ & $91.12 \pm 12.01$ \\
\hline
\end{tabular}

Note: The fitted and removed from the time series data model comprises the order polynomial and a sum of complex sinusoids with periods $365.25,180.0,120.0$ days. The phase in degrees is given with respect to the reference epoch 51544.5 (1 January 2000).
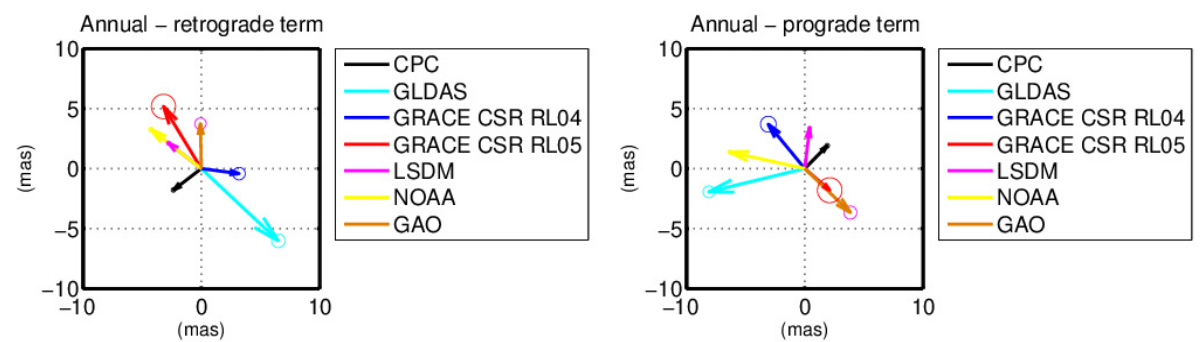

Fig. 7. Phasor diagrams of annual variations of the complex-valued component $\chi_{1}+i \chi_{2}$ of regional hydrological and gravimetric excitation functions and the geodetic residuals GAM-NCEP/NCAR-ECCO. Analysis was done over the period of 2003.0-2006.0.

The differences between the geodetic residuals, however, make it difficult to find the hydrological and gravimetric excitation function which is closest to the signal obtained from the geodetic observations. Here for further analysis we chose the geodetic residuals GAM-NCEP/NCAR-ECCO which have the smallest values of the standard deviations. The comparison of GAM-NCEP/NCAR-ECCO and HAM Global was carried out in two ways: by determining the annual oscillation parameters (see Table 6 and Fig. 7) and by computing the correlation coefficients of non-seasonal variations of the series obtained by subtracting the seasonal signals model (365, 
181 , and 121 days) from the time series (Table 4). Relatively good agreement of the vectors exists for the GRACE RL05 case.

The calculated correlation coefficients indicate that there is better agreement between the $\chi_{2}$ components than between the $\chi_{1}$ components (Table 5). The highest value, equal to 0.53 , of the correlation coefficient was reached when the CSR RL05 data were used to estimate the gravimetric computation.

\section{CONCLUSIONS}

An inter-comparison analysis was conducted of various hydrological and gravimetric excitation functions of polar motion using different models of land hydrology and data from the GRACE mission.

The results indicate that the regional hydrological and gravimetric excitation functions of polar motion of the $\chi_{2}$ component show changes in larger land areas than the regional hydrological and gravimetric excitation functions of polar motion of the $\chi_{1}$ component. Thus, changes in continental water storage play a more significant role in polar motion in $\chi_{2}$ than in the $\chi_{1}$ component. This fact can be explained as being due to the geographical locations of the major continents aligning closer along the $\mathrm{Y}$ axis. Polar motion $\mathrm{Y}$ is more sensitive to mass changes over land and $\mathrm{X}$ is more sensitive to mass changes over the ocean.

The maximum values of the hydrological and gravimetric excitation of polar motion, determined from five different hydrological models of land hydrology and from the two series of GRACE data, have shown similar geographical patterns, but the amplitudes of these variations are different.

The studies show that the maximum values of regional hydrological and gravimetric excitation functions are in the following regions: Southeast and South Asia, the Amazon basin of South America, the southeast United States and areas north of the Mediterranean Sea, which is in agreement with Nastula et al. (2008b) and Nastula and Salstein (2012). Additionally, these studies have shown that the GRACE regional excitation functions of polar motion have larger magnitudes than the regional hydrological excitation functions computed from the hydrological models.

The time series of HAM determined for the five selected land regions of maximum variability are dominated by annual oscillation. The largest amplitudes of $\chi_{1}$ are for the European region; and for the $\chi_{2}$ components they are for the Asian region. The smallest amplitudes of the $\chi_{1}$ components are for the North American regions, and the $\chi_{2}$ components are for the African regions. The modelled hydrological and gravimetric time series of the $\chi_{1}$ components for the European region varied from the mean on the 3-8 mas level, apart from the time series based on the LSDM model and GRACE CSR 
RL05 data. The time series of the $\chi_{2}$ components for the Asian region varied from the mean on the 5-9 mas level.

It should be emphasized that the areas where the variations of HAM were relatively small on the maps make an important contribution to the HAM Global function.

Comparisons of geodetic residuals show significant differences among them. These large differences (between 10 and 20 mas) occur between geodetic residuals determined using different oceanic models. In contrast, the use of different atmospheric models does not cause any significant difference in the geodetic residuals. This result shows that the ocean's contribution to geophysical excitation functions of polar motion is still less determinate than the influence of the atmosphere.

The comparison of geodetic residuals and global hydrological and gravimetric excitation functions of polar motion shows that the amplitudes of HAM variations are smaller than the variations of geodetic residuals. Therefore, the HAM hydrological and gravimetric excitation functions do not have enough energy to significantly improve agreement between geodetic excitation and geophysical (AMM+OAM+HAM) ones. Despite the large number of differences between the hydrological and gravimetric excitation functions, their contribution to polar motion excitation is not negligible and cannot be rejected in the analysis.

Further studies on geophysical excitation of the observed polar motion should focus on improving the OAM and HAM estimations. Analysis of regional changes in these functions may be helpful in improving their models.

Acknowledgements. This work was supported by the Polish national science foundation NCN under grant No. DEC-2012/05/B/ST10/02132.

\section{References}

Bizouard, C., and D. Gambis (2009), The combined solution C04 for Earth orientation parameters consistent with international terrestrial reference frame 2005. In: H. Drewes (ed.), Geodetic Reference Frames, IAG Symposium, Munich, Germany, October 9-14, 2006, Springer, Berlin Heidelberg, 265270, DOI: 10.1007/978-3-642-00860-3_41.

Brzeziński, A. (1992), Polar motion excitation by variations of the effective angular momentum function: considerations concerning deconvolution problem, Manuscr. Geod. 17, 1, 3-20.

Brzeziński, A., J. Nastula, B. Kołaczek, and R.M. Ponte (2005), Oceanic excitation of polar motion from intraseasonal to decadal periods. In: F. Sansõ (ed.), 
A Window of the Future of Geodesy, IAG Symposium, Sapporo, Japan, June 30 - July 11, 2003, Springer, Berlin Heidelberg, 591-596, DOI: 10.1007/3-540-27432-4_100.

Brzeziński, A., J. Nastula, and B. Kołaczek (2009), Seasonal excitation of polar motion estimated from recent geophysical models and observations, J. Geodyn. 48, 3-5, 235-240, DOI: 10.1016/j.jog.2009.09.021.

Chambers, D.P., and J.A. Bonin (2012), Evaluation of Release-05 GRACE timevariable gravity coefficients over the ocean, Ocean Sci. 8, 5, 859-868, DOI: 10.5194/os-8-859-2012.

Chao, B.F., and R.S. Gross (1987), Changes in the Earth's rotation and low-degree gravitational field induced by earthquakes, Geophys. J. Int. 91, 3, 569-596, DOI: 10.1111/j.1365-246X.1987.tb01659.x.

Chao, B.F., and W.P. O'Connor (1988), Global surface-water-induced seasonal variations in the Earth's rotation and gravitational field, Geophys. J. Int. 94, 2, 263-270, DOI: 10.1111/j.1365-246X.1988.tb05900.x.

Chen, J.L., and C.R. Wilson (2005), Hydrological excitations of polar motion 19932002, Geophys. J. Int. 160, 3, 833-839, DOI: 10.1111/j.1365-246X.2005. 02522.x.

Chen, J.L., C.R. Wilson, B.F. Chao, C.K. Shum, and B.D. Tapley (2000), Hydrological and oceanic excitations to polar motion and length-of-day variation, Geophys. J. Int. 141, 1, 149-156, DOI: 10.1046/j.1365-246X.2000.00069.x.

Dill, R., M. Thomas, and C. Walter (2009), Hydrological induced Earth rotation variations from stand-alone and dynamically coupled simulations. In: M. Soffel and N. Capitaine (eds.), Proc. Journées 2008 "Systèmes de Référence Spatio-temporels" and X. Lohrmann-Kolloquium, 22-24 September 2008, Dresden, Germany, 115-118.

Dobslaw, H., R. Dill, A. Grötzsch, A. Brzeziński, and M. Thomas (2010), Seasonal polar motion excitation from numerical models of atmosphere, ocean, and continental hydrosphere, J. Geophys. Res. 115, B10, B10406, DOI: 10.1029/2009JB007127.

Eubanks, T.M. (1993), Variations in the orientation of the Earth. In: D.E. Smith and D.L. Turcotte (eds.), Contributions of Space Geodesy to Geodynamics: Earth Dynamics, American Geophysical Union, Washington, 1-54, DOI: 10.1029/GD024p0001.

Fan, Y., and H. van den Dool (2004), Climate Prediction Center global monthly soil moisture data set at 0.5 degrees resolution for 1948 to present, J. Geophys. Res. 109, D10, D10102, DOI: 10.1029/2003JD004345.

Gross, R.S. (2005), The observed period and Q of the Chandler wobble. In: H.-P. Plag, B.F. Chao, R.S. Gross, and T. van Dam (eds.), Forcing of Polar Motion in the Chandler Frequency Band: A Contribution to Understanding Interannual Climate Variations. Proceedings of the Workshop Held April 21-23, 2004 at the Hotel Parc Belle-Vue, Luxembourg, Centre Européen de Géodynamique et de Séismologie, Luxembourg, 31-37. 
Gross, R.S., I. Fukumori, and D. Menemenlis (2003), Atmospheric and oceanic excitation of the Earth's wobbles during 1980-2000, J. Geophys. Res. 108, B8, 2370, DOI: 10.1029/2002JB002143.

Hinnov, L.A., and C.R. Wilson (1987), An estimate of the water storage contribution to the excitation of polar motion, Geophys. J. Int. 88, 2, 437-459, DOI: 10.1111/j.1365-246X.1987.tb06652.x.

Höpfner, J. (2001), Atmospheric, oceanic and hydrological contributions to seasonal variations in length of day, $J$. Geodesy 75, 2-3, 137-150, DOI: 10.1007/ s001900100164.

Jin, S.G., D.P. Chambers, and B.D. Tapley (2010), Hydrological and oceanic effects on polar motion from GRACE and models, J. Geophys. Res. 115, B2, B02403, DOI: 10.1029/2009JB006635.

Jin, S.G., L.J. Zhang, and B.D. Tapley (2011), The understanding of length-of-day variations from satellite gravity and laser ranging measurements, Geophys. J. Int. 184, 2, 651-660, DOI: 10.1111/j.1365-246X.2010.04869.x.

Jin, S.G., A.A. Hassan, and G.P. Feng (2012), Assessment of terrestrial water contributions to polar motion from GRACE and hydrological models, J. Geodyn. 62, 40-48, DOI: 10.1016/j.jog.2012.01.009.

Kalnay, E., M. Kanamitsu, R. Kistler, W. Collins, D. Deaven, L. Gandin, M. Iredell, S. Saha, G. White, J. Woollen, Y. Zhu, A. Leetmaa, R. Reynolds, M. Chelliah, W. Ebisuzaki, W. Higgins, J. Janowiak, K.C. Mo, C. Ropelewski, J. Wang, R. Jenne, and D. Joseph (1996), The NCEP/NCAR 40-year reanalysis project, Bull. Am. Meteorol. Soc. 77, 3, 437-471, DOI: 10.1175/ 1520-0477(1996)077<0437:TNYRP $>2.0$. CO;2.

Kołaczek, B., J. Nastula, and M. Paśnicka (2011), Analyses of the geophysical excitations of polar motion in the period: 2001.0-2009.0. In: H. Schuh, S. Boehm, T. Nilsson, and N. Capitaine (eds.), Proc. Journees 2011 "Systemes de Reference Spatio-temporels", Vienna University of Technology, 19-21 September 2011, Vienna, Austria.

Kuehne, J., and C.R. Wilson (1988), Chandler wobble excitation by terrestrial water storage, Eos Trans. AGU 69, 16, 328, DOI: 10.1029/EO069i016p00299.

Kuehne, J., and C.R. Wilson (1991), Terrestrial water storage and polar motion, J. Geophys. Res. 96, B3, 4337-4345, DOI: 10.1029/90JB02573.

Lettenmaier, D.P., and J.S. Famiglietti (2006), Hydrology: Water from on high, $\mathrm{Na}$ ture 444, 7119, 562-563, DOI: 10.1038/444562a.

Nastula, J. (1997), The regional atmospheric contributions to the polar motion and EAAM excitation functions. In: J. Segawa, H. Fujimoto, and S. Okubo (eds.), Gravity, Geoid and Marine Geodesy, International Symposium, Tokyo, Japan, September 30 - October 5, 1996, 281-288, DOI: 10.1007/9783-662-03482-8_39.

Nastula, J., and R.M. Ponte (1999), Further evidence of oceanic excitation of polar motion, Geophys. J. Int. 139, 1, 123-130, DOI: 10.1046/j.1365-246X.1999. 00930.x. 
Nastula, J., and D.A. Salstein (2012), Regional geophysical excitation functions of polar motion over land areas. In: S. Kenyon, M.C. Pacino, and U. Marti (eds.), Geodesy for Planet Earth, Proc. of the 2009 IAG Symposium, Buenos Aires, Argentina, 31 August - 4 September 2009, Part 3, 491-497, DOI: 10.1007/978-3-642-20338-1_59.

Nastula, J., R.M. Ponte, and D.A. Salstein (2000), Regional signals in atmospheric and oceanic excitation of polar motion. In: S. Dick, D. McCarthy, and B. Luzum (eds.), Polar Motion: Historical and Scientific Problems, ASP Conference Series, Vol. 208, Astronomical Society of the Pacific, San Francisco, 463-472.

Nastula, J., B. Kołaczek, and D.A. Salstein (2008a), Regional differences of hydrological excitation of polar motion computed from hydrological models and from the GRACE gravity field data, Geophys. Res. Abstr. 10, EGU2008-A08451.

Nastula, J., B. Kołaczek, and D. Salstein (2008b), Comparison of regional hydrological excitation of polar motion derived from hydrological models and the GRACE gravity field data. In: M. Soffel and N. Capitaine (eds.), Proc. Journées 2008 "Systèmes de Référence Spatio-temporels" and X. Lohrmann-Kolloquium, 22-24 September 2008, Dresden, Germany.

Nastula, J., D. Salstein, and B. Kołaczek (2009), Patterns of atmospheric excitation functions of polar motion from high-resolution regional sectors, J. Geophys. Res. 114, B4, B04407, DOI: 10.1029/2008JB005605.

Nastula, J., M. Paśnicka, and B. Kołaczek (2011), Comparison of the geophysical excitations of polar motion from the period: 1980.0-2009.0, Acta Geophys. 59, 3, 561-577, DOI: 10.2478/s11600-011-0008-2.

Ponte, R.M., D. Stammer, and J. Marshall (1998), Oceanic signals in observed motions of the Earth's pole of rotation, Nature 391, 6666, 476-479, DOI: $10.1038 / 35126$.

Rodell, M., P.R. Houser, U. Jambor, J. Gottschalck, K. Mitchell, C.-J. Meng, K. Arsenault, B. Cosgrove, J. Radakovich, M. Bosilovich, J.K. Entin, J.P. Walker, D. Lohmann, and D. Toll (2004), The global land data assimilation system, Bull. Amer. Meteorol. Soc. 85, 381-394, DOI: 10.1175/ BAMS-85-3-381.

Salstein, D.A., and R.D. Rosen (1989), Regional contributions to the atmospheric excitation of rapid polar motions, J. Geophys. Res. 94, D7, 9971-9978, DOI: 10.1029/JD094iD07p09971.

Salstein, D.A., R.D. Rosen, D.M. Kann, and A.J. Miller (1993), The sub-bureau for atmospheric angular momentum of the International Earth Rotation Service: A meteorological data center with geodetic applications, Bull. Am. Meteorol. Soc. 74, 1. 67-80, DOI: 10.1175/1520-0477(1993)074<0067: TSBFAA $>2.0 . \mathrm{CO} ; 2$. 
Seaone, L., J. Nastula, C. Bizouard, and D. Gambis (2009), The use of gravimetric data from GRACE mission in the understanding of polar motion variations, Geophys. J. Int. 178, 2, 614-622, DOI: 10.1111/j.1365-246X.2009.04181.x.

van Hylckama, T.E.A. (1970), Water balance and Earth unbalance. In: Proc. Reading Symp. World Water Balance, International Associations of Scientific Hydrology, Vol. 92, AIHS-UNESCO Publication, 434-444.

Wahr, J., and M. Molenaar (1998), Time variability of the Earth's gravity field: Hydrological and oceanic effects and their possible detection using GRACE, J. Geophys. Res. 103, B12, 30205-30229, DOI: 10.1029/98JB02844.

Walter, C. (2008), Simulationen hydrologischer Massenvariationen und deren Einfluss auf die Erdrotation, Ph.D. Thesis, Technischen Universität, Dresden, Germany, 195 pp.

Received 9 February 2015

Received in revised form 27 May 2015

Accepted 23 June 2015 